\title{
Arterial Phase Spiral CT
}

National Cancer Institute

\section{Source}

National Cancer Institute. Arterial Phase Spiral CT. NCI Thesaurus. Code C19910.

A type of computed tomog raphy in which images are taken during the time when the contrast medium has passed the heart and entered the arteries. 